\title{
COMPUTATIONAL PERFORMANCE OF QUANTUM PHASE ESTIMATION ALGORITHM
}

\author{
Zhuang Jiayu ${ }^{1}$, Zhao Junsuo and Xu Fanjiang \\ ${ }^{1}$ Science and Technology on Integrated Information System Laboratory, \\ Institute of Software Chinese Academy of Sciences, Beijing, China \\ jiayuliscas.ac.cn
}

\begin{abstract}
A quantum computation problem is discussed in this paper. Many new features that make quantum computation superior to classical computation can be attributed to quantum coherence effect, which depends on the phase of quantum coherent state. Quantum Fourier transform algorithm, the most commonly used algorithm, is introduced. And one of its most important applications, phase estimation of quantum state based on quantum Fourier transform, is presented in details. The flow of phase estimation algorithm and the quantum circuit model are shown. And the error of the output phase value, as well as the probability of measurement, is analysed. The probability distribution of the measuring result of phase value is presented and the computational efficiency is discussed.
\end{abstract}

\section{KEYWORDS}

Quantum computation, Quantum Fourier transform, Phase estimation

\section{INTRODUCTION}

In the past few decades, we have gained more and more ability to access massive amounts of information and to make use of computers to store, analyse and manage this data. Recent studies have shown the great progress towards the physical realization of a quantum computer. Deutsch ${ }^{[1]}$ ${ }^{[2]}$ systematically described the first universal quantum computer that is accepted by now. In 1982, Benioff $^{[3]}$ studied the question whether quantum computer was more computationally powerful than a conventional classical Turing machine. He mapped the operation of a reversible Turing machine onto the quantum system and thus exhibited the first quantum-mechanical model of computation, which discovered the potential power of quantum computer. In 1994, American scientist Peter Shor ${ }^{[4]}$ proposed an algorithm that factor a large integer in polynomial time, which is the first practical quantum algorithm. In 1996, Grover ${ }^{[5][6]}$ proposed an algorithm that provides a speedup of $\sqrt{N}$ in order of magnitude than classic algorithm in searching an unsorted database. The algorithm caused attention as its potential of solving NP problem. Quantum computation and quantum information is the study of the information processing task that can be accomplished using quantum mechanical system. Quantum computation has many features that differ from classical computing in that quantum state has the characteristic of coherence and entanglement.

\section{THE QUANTUM Fourier TRANSFORM (QFT)}

One of the most useful methods of solving problems in mathematics or computer science is to transform it into some other problem for which a solution is known. The quantum Fourier transform $^{[7]}$ is a kind of discrete Fourier transform which plays an important role in many

Sundarapandian et al. (Eds) : CSE, CICS, DBDM, AIFL, SCOM - 2013

pp. 23-27, 2013. (C) CS \& IT-CSCP 2013

DOI : $10.5121 /$ csit.2013.3303 
quantum algorithms. For example, Shor's algorithm, order finding algorithm and hidden subgroup problem.

Quantum Fourier Transform is defined as follow: $\mathrm{U}_{\mathrm{QFT}}|\mathrm{x}\rangle=\frac{1}{\sqrt{2^{\mathrm{n}}}} \sum_{\mathrm{y}=0}^{2^{\mathrm{n}}-1} \mathrm{e}^{\frac{2 \pi \mathrm{ixy}}{2^{\mathrm{n}}}}|\mathrm{y}\rangle$. The QFT is a unitary operator in $2^{\mathrm{n}}$-dimensional vector space, it is defined as a linear operator in a group of orthonormal base $|0>| 1>,\cdots \mid 2^{n}-1>$. Since any quantum computation algorithm in an n-qubit quantum computer is based on operations by matrices in $\mathrm{U}\left(2^{\mathrm{n}}\right.$-dimensional), in this sense we have the universality of the QFT. In the QFT, we used the following basic quantum gates: Hadmard gate and Controlled-Phase Shift gate. Hadmard gate $(\mathrm{H})$ is acts on one qubit. Controlled-Phase Shift gate $\left(\mathrm{U}_{\mathrm{j}, \mathrm{k}}\right)$ is acts on two qubit, $\mathrm{k}$ is control qubit and $\mathrm{j}$ is target qubit. Applying the unitary transformation $\mathrm{U}_{\mathrm{j}, \mathrm{k}}$ on the $\mathrm{j}^{\text {th }}$ qubit if and only if the $\mathrm{k}^{\text {th }}$ qubit is $\mid 1>$. The transform matrixes of the gates operator in Hilbert space are presented as follow: (Phase shift $\left.\theta_{\mathrm{j}, \mathrm{k}}=\frac{2 \pi \mathrm{x}_{\mathrm{k}}}{2^{\mathrm{k}-\mathrm{j}+1}}\right)$

$$
\mathrm{H}=\frac{1}{\sqrt{2}}\left[\begin{array}{cc}
1 & 1 \\
1 & -1
\end{array}\right] \quad \mathrm{U}_{\mathrm{j}, \mathrm{k}}=\left[\begin{array}{cccc}
1 & 0 & 0 & 0 \\
0 & 1 & 0 & 0 \\
0 & 0 & 1 & 0 \\
0 & 0 & 0 & \mathrm{e}^{\mathrm{i} \theta_{\mathrm{j}, \mathrm{k}}}
\end{array}\right]
$$

The QFT can be given the following useful product representation:

$$
\mathrm{U}_{\mathrm{QFT}}\left|\mathrm{x}_{1} \cdots \mathrm{x}_{\mathrm{n}-1} \mathrm{x}_{\mathrm{n}}\right\rangle=\frac{1}{\sqrt{2^{\mathrm{n}}}}\left(|0\rangle+\mathrm{e}^{2 \pi \mathrm{i} 0 \cdot \mathrm{x}_{\mathrm{n}}}|1\rangle\right) \cdots\left(|0\rangle+\mathrm{e}^{2 \pi \mathrm{i} 0 \cdot \mathrm{x}_{1} \cdots \mathrm{x}_{\mathrm{n}-1} \mathrm{x}_{\mathrm{n}}}|1\rangle\right)
$$

Most of the properties of the quantum Fourier transform follow from the fact that it is a unitary transformation. From the unitary property it follows that the inverse of the quantum Fourier transform is the Hermitian adjoint of the Fourier matrix, therefore, $\mathrm{U}_{\mathrm{QFT}}{ }^{-1}=\mathrm{U}_{\mathrm{QFT}}{ }^{\dagger}$. Since there is an efficient quantum circuit implementing the quantum Fourier transform, the circuit can be run in reverse to perform the inverse quantum Fourier transform. Thus both transforms can be efficiently performed on a quantum computer. The inverse of QFT is : $\mathrm{U}_{\mathrm{QFT}}^{\dagger}|\mathrm{x}\rangle=\frac{1}{\sqrt{2^{\mathrm{n}}}} \sum_{\mathrm{y}=0}^{2^{\mathrm{n}}-1} \mathrm{e}^{-\frac{2 \pi \mathrm{i} \cdot \mathrm{xy}}{2^{\mathrm{n}}}}|\mathrm{y}\rangle$.

The quantum circuit representation of the QFT is show in Figure1.

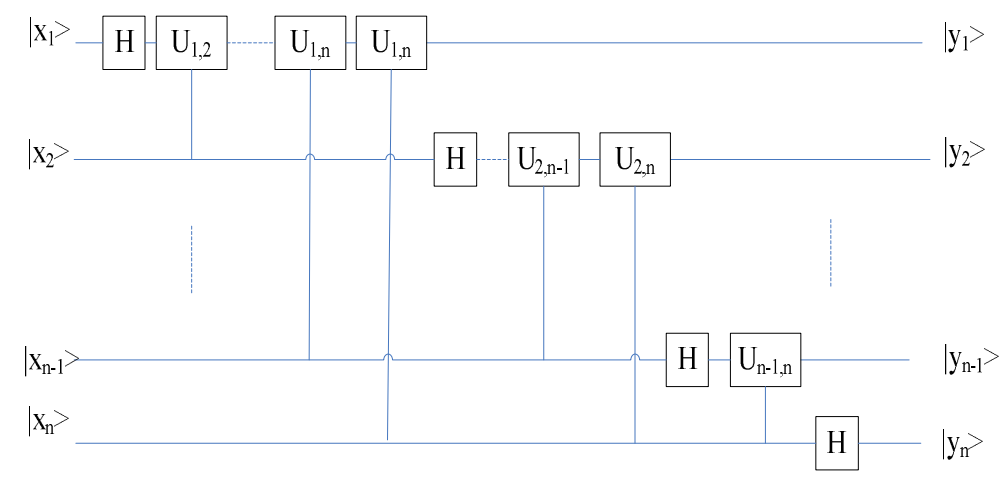

Figure 1. Circuit of the quantum Fourier transform 


\section{Phase estimation And Simulation}

\subsection{Phase estimation}

Suppose, there is an unitary operator has an eigenvector $|a\rangle$ with eigenvalue $\mathrm{e}^{2 \pi \mathrm{i} \varphi}$ (n-qubit), $\mathrm{U}|\mathrm{a}\rangle=\mathrm{e}^{2 \pi \mathrm{i} \varphi}|\mathrm{a}\rangle$. The quantum phase estimation ${ }^{[8]}$ procedure uses two registers. The first register with $\mathrm{m}$-qubit, it used to store the value of phase $\mid \varphi_{1} \varphi_{2} \cdots \varphi_{\mathrm{m}}>\left(\varphi_{\mathrm{i}}=0\right.$ or $\left.1, \mathrm{i}=1,2 \ldots \mathrm{m}\right)$, in decimal it equals to $\sum_{\mathrm{i}=1}^{\mathrm{m}} \frac{\varphi_{\mathrm{i}}}{2^{\mathrm{i}}}$. The error of true value and store value is $\mu$ which can be expressed: $\varphi=\left(\sum_{\mathrm{i}=1}^{\mathrm{m}} \frac{\varphi_{\mathrm{i}}}{2^{\mathrm{i}}}\right)+\mu \quad\left(|\mu| \leq \frac{1}{2^{\mathrm{m}+1}}\right)$. The first register initially in the state $|0 \cdots 0\rangle$ while the second is $|\mathrm{a}\rangle$. Apply the quantum circuit shown in Figure 2. $\left(U_{\mathrm{n}}=U^{2^{n}}\right)$

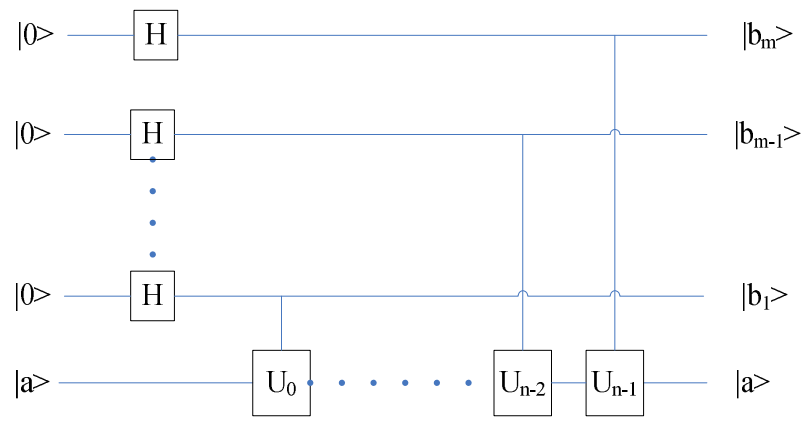

Figure 2. First stage of the phase estimation

Apply the inverse quantum Fourier transform on the first register $\left(\left|b_{1} \cdots b_{m-1} b_{m}\right\rangle\right)$, we have the state:

$$
\frac{1}{2^{n}} \sum_{j=0}^{2^{n}-1} \sum_{k=0}^{2^{n}-1} e^{\frac{-2 \pi i j k}{2^{n}}} \mathrm{e}^{2 \pi \mathrm{i} \varphi \mathrm{j}}|\mathrm{k}\rangle
$$

And then, read out the state of first register by doing a measurement in the computation basis, the result is that $\widetilde{\varphi}=0 . \varphi_{1} \cdots \varphi_{m}$ which is an estimator for $\varphi$.

\subsection{Simulation}

We proposed a quantum computation emulator ${ }^{[9]}[10]$ in classical computer which satisfies the requirements of quantum computer. Conventional quantum algorithms can be operated in this simulation system. In this paper, the simulation aimed to analyses the relationship between the accuracy and probability of observing in phase estimation algorithm. The phase can be written in $\mathrm{n}$-qubit in the phase estimation algorithm. We will get all the possible states by measuring the output qubit.

In the first simulation, we provide 8 -qubit for the phase estimation. It means the minimum accuracy is $1 / 256$. Suppose the phase is $1 / 3$. We can get the probability distributions of quantum state of output from $|0 \cdots 0\rangle$ to $|1 \cdots 1\rangle$ which is shown in Figure 3. 


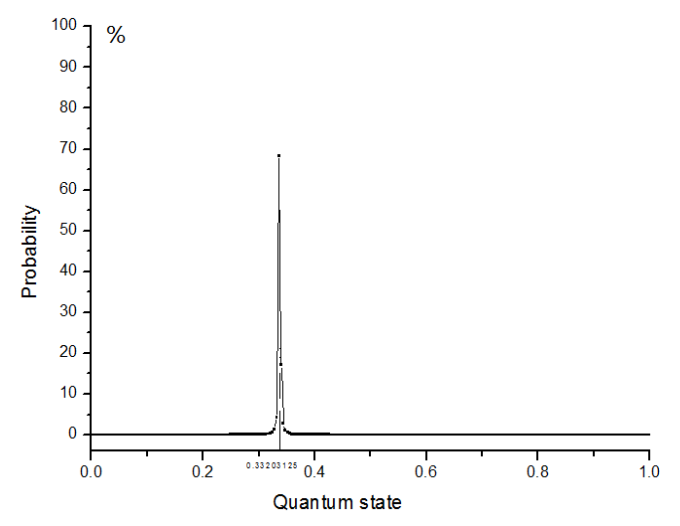

Figure 3. The probability distribution of phase estimation

The second simulation is mainly about the different numbers of qubit effect on the probability of phase estimation which in the same accuracy. Suppose the phase is $1 / 3$ and we wish to approximate the phase to an accuracy 1/64. And then we provide different number of qubit for the phase estimation. In Figure 4, (a) is the probability of success estimation by different number of qubit and (b) is the time steps by different number of qubit.

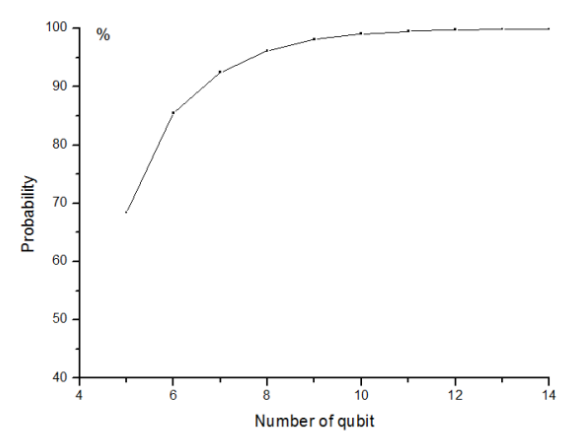

(a)

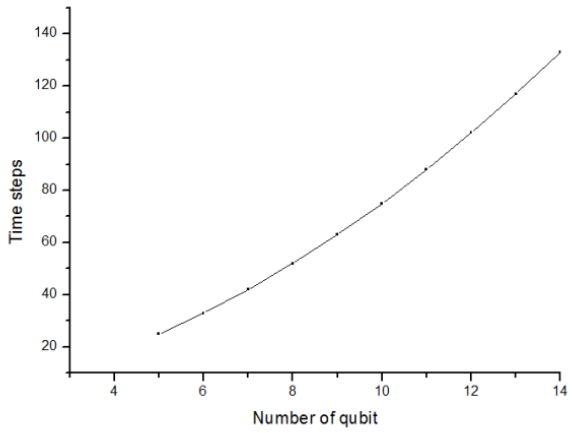

(b)

Figure 4. The probability and time steps by different number of qubit

\section{Conclusions}

This paper describes the phase estimation algorithm based in quantum Fourier transform. And the algorithm was operated in a simulation system. In Figure3, the result converged to the expectation value and it proved the phase estimation algorithm is an efficient estimate. In Figure4, we can see the probability increased with the number of qubit with given accuracy, but the probability changes slowly when the number of qubit reaches a certain value. Meanwhile, the time steps were increased in polynomial multiple. We should design appropriate number of qubit for the phase estimation with high success probability and low cost. In future, our study focuses on the optimization methods in quantum computation. 


\section{REFERENCES}

[1] Deutsch D. Quantum theory, the Church-Turing principle and the universal quantum computer[J]. Proc. Royal Soc. Lond, vol, A400, 1985, pp, 97-117

[2] Deutsch D. Quantum computational networks, Proc. Roy. Soc. London, A. 439(1992)553-558

[3] Benioff P. The computer as a physical stystem: a microscopic quantum mechanical Hamiltonian model of computers as represented by Turing machines. Journal of Statistical Physics, 1982,22:563591

[4] Shor P.W. , Algorithms for Quantum Computation: Discrete Logarithms and Factoring, 35th Annual Symposium on Foundations of Computer Science, New Mexico: IEEE Computer Society Press, 1994, 124 134

[5] Grover L K. A fast quantum mechanical algorithm for database search. Proceeding 28th Annual ACM symposium on the theory of computing, 1996, 212-129

[6] Grover L K. Quantum mechanics helps in searching for a needle in haystack[J]. Phys Rev Lett, 1997,79:325-328

[7] Nilelsen M. A. \& Chuang L.L. Quantum computation and Quantum Information[M]. Cambridge Univ. Press 2000.

[8] B.C.Sanders \& G.J.Milburn Optimal quantum measurements for phase estimation, Phys. Rev. Lett. 75, 1995, 2944-2947

[9] Feynman R. Quantum mechanical computers[J]. Science, 1996, vol. 273. No.5278, pp. 1073-1078

[10] I.Buluta, F.Nori Quantum simulators[J]. Science, 2009, vol. 326. No.5949, pp. 108-111

\section{Authors}

Zhuang Jiayu is a researcher in Institute of Software Chinese Academy of Sciences. The author interested in the research of quantum computation and quantum ciruits.

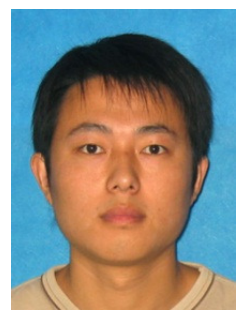

